\title{
Exact analytical evaluation of time dependent transmission coefficient from the method of reactive flux for an inverted parabolic barrier
}

\author{
Rajarshi Chakrabarti \\ Department of Inorganic and Physical Chemistry, \\ Indian Institute of Science, Bangalore, 560012, India
}

\begin{abstract}
In this paper we derive a general expression for the transmission coefficient using the method of reactive flux for a particle coupled to a harmonic bath surmounting a one dimensional inverted parabolic barrier. Unlike Kohen and Tannor [J. Chem. Phys. 103, 6013 (1995)] we use a normal mode analysis where the unstable and the other modes have a complete physical meaning. Importantly our approach results a very general expression for the time dependent transmission coefficient not restricted to overdamped limit. Once the spectral density for the problem is known one can use our formula to evaluate the time dependent transmission coefficient. We have done the calculations with time dependent friction used by Xie [Phys. Rev. Lett 93, 180603 (2004)] and also the one used by Kohen and Tannor [J. Chem. Phys. 103, 6013 (1995)]. Like the formula of Kohen and Tannor our formula also reproduces the results of transition state theory as well as the Kramers theory in the limits $t \rightarrow 0$ and $t \rightarrow \infty$ respectively.
\end{abstract}

\section{INTRODUCTION}

The dynamics of a particle surmounting a barrier is an important and interesting problem in chemistry and physics. In chemistry, chemical reactions are most common examples where one studies the dynamics of barrier crossing along a suitably defined reaction coordinate. While in physics nucleation phenomena, electrical transport etc. involve barrier crossing. A review by Hänggi, Talkner and Borkovec [1] gives a comprehensive account on the subject of barrier crossing. The simplest possible approach to calculate the rate constant for such processes is given by transition state theory [1, 2]. As the simplest version of the problem one can describe the rate process along a single reaction co-ordinate. Then the one dimensional version of the transition state rate constant $k_{T S T}$ reads as, $k_{T S T}=\frac{\omega_{0}}{2 \pi} \exp \left[-\frac{E_{b}}{k_{B} T}\right]$, where $\omega_{0}$ is the angular frequency in the reactant well and $E_{b}$ is the barrier height or the activation energy. The derivation of transition state rate constant is based on two assumptions. Firstly an overall thermal equilibrium is assumed and secondly frictional effect is not taken into account by incorporating what is known as "no recrossing" assumption. Subsequently, Kramers [1, 2, 3, 4] theory takes into account of the friction in a phenomenological way. His formulation was based on Markovian dynamics of a Brownian particle escaping from a metastable state. Further extensions of Kramers's theory were carried out by Grote and Hynes [5] followed by Hänggi and Mojtabi [6]. They took into account of the non-Markovian nature of the dynamics. Another approach to calculate the rate constant is due to Chandler [7, 8] known as the method of reactive flux in chemical physics literature. In this approach the thermal rate constant is written as a correlation function which is nothing but the ensemble average over infinite number of trajectories starting at the barrier top and ending on the product side at time $t$. The rate constant at time $t$ is written as a product of the time dependent transmission coefficient $\kappa(t)$ and the transition state rate constant $k_{T S T}$. Thus one writes, $k(t)=\kappa(t) k_{T S T}$. In the limit $t \rightarrow 0, \kappa(t) \rightarrow 1$ and one obtains $k(0)=k_{T S T}$. Here we follow the method of reactive flux to calculate the time dependent rate constant for a particle bilinearly coupled to a harmonic bath [9, 10, 11]. The bilinear coupling allows us to get an analytical expression for the time dependent transmission coefficient. Although the method of reactive flux and the hamiltonian we have used are well known in the literature, as far as our knowledge nobody has used such hamiltonian to calculate the time dependent transmission coefficient analytically. The calculation is of interest as the transmission coefficient for the overdamped limit of the model was obtained recently by simulation [12]. Our approach directly gives expression for the time dependent transmission coefficient in terms of the spectral density $J(\omega)$ and not restricted to overdamped limit. A normal mode analysis of the coupled Hamiltonian was made by Pollak [13, 14, 15] and others to analyze the Kramers problem. Here we point out that the approximation is very powerful and can be used to get the exact expression for $\kappa(t)$. Further we believe that it can also be used to get the quantum expression for $\kappa(t)$. An exact calculation of $\kappa(t)$ using the phase space distribution function formulation was carried out in classical regime by Kohen and Tannor [16, 17], Bao [18], and more recently in the context of single enzyme kinetics by Chowdhury and Cherayil [19]. Also the calculation of the transmission coefficient in the quantum domain is carried out by Ray's group [20]. Our analysis in terms of normal modes has the advantage that it makes the physical ideas very clear. The paper is arranged as follows. In section II we introduce our system plus harmonic bath hamiltonian and the Generalized Langevin Equation (GLE). In section III we mention the method of reactive flux and using our hamiltonian derive an analytical expression for the time dependent transmission coefficient, $\kappa(t)$. Section IV deals with the calculation of $\kappa(t)$ for Markovian and Non Markovian dynamics. Section V is conclusion. 


\section{GENERALIZED LANGEVIN EQUATION AND NORMAL MODE ANALYSIS}

We consider a particle coupled to a harmonic bath 9 , 10] with the total hamiltonian

$H=\frac{P^{2}}{2 M}+V(Q)+\frac{1}{2} \sum_{j=1}^{N}\left(\frac{P_{j}^{2}}{m_{j}}+m_{j} \omega_{j}^{2}\left(Q_{j}-\frac{c_{j}}{m_{j} \omega_{j}} Q\right)^{2}\right)$

Here $\left(P_{j}, Q_{j}\right)$ are the momenta and coordinates of the $j$ th bath oscillator whose mass and frequency are $m_{j}, \omega_{j}$ respectively. $(P, Q)$ is the momentum and coordinate of the system. $c_{j}$ couples the bath oscillator to the system. Now in terms of the mass weighted coordinates

$$
q=\sqrt{M} Q, q_{j}=\sqrt{m_{j}} Q_{j}
$$

our Hamiltonian becomes

$$
H=\frac{p^{2}}{2}+V(q)+\frac{1}{2} \sum_{j=1}^{N}\left(p_{j}^{2}+\omega_{j}^{2}\left(q_{j}-\frac{c_{j}}{\sqrt{M m_{j}}} q\right)^{2}\right)
$$

and then with this Hamiltonian one can derive what is know as the Generalized Langevin Equation (GLE) which reads as

$$
\ddot{q}(t)+V^{\prime}(q)+\int_{0}^{t} d t^{\prime} \gamma\left(t-t^{\prime}\right) \dot{q}\left(t^{\prime}\right)=\zeta(t)
$$

One can also write the time dependent friction in terms of the spectral density of the bath $J(\omega)$ as follows

$$
\gamma(t)=\frac{\Theta(t)}{M} \frac{2}{\pi} \sum_{j=1}^{N} \int_{0}^{\infty} d \omega \frac{J(\omega)}{\omega} \cos (\omega t)
$$

where

$$
J(\omega)=\frac{\pi}{2} \sum_{j=1}^{N} \frac{c_{j}^{2}}{m_{j} \omega_{j}} \delta\left(\omega-\omega_{j}\right)
$$

One can easily show that

$$
\left\langle\zeta\left(t_{1}\right) \zeta\left(t_{2}\right)\right\rangle=M k_{B} T \gamma\left(t_{1}-t_{2}\right)
$$

We take the barrier top to be at $q=0$ and introduce $\omega_{b}^{2}=\left(\frac{\partial^{2} V}{\partial q^{2}}\right)_{q=0}$. For small amplitude motion around the barrier top, one can use the normal modes, $\eta_{j}=\sum_{i=0}^{N} U_{j i} q_{i}$ where $\underline{\underline{U}}$ is an orthogonal matrix such that $\underline{\underline{U}} \underline{\underline{D}} \underline{\underline{U}}^{T}$ is diagonal. $\underline{\underline{D}}$ is a dynamical matrix given by

$$
\underline{\underline{D}}=\left(\begin{array}{cccc}
-\omega_{b}^{2}+\sum_{j=1}^{N} \frac{c_{j}^{2}}{M m_{j} \omega_{j}^{2}} & \cdot & \cdot & -\frac{c_{j}}{\sqrt{M m_{j}}} \\
\cdot & \cdot & \cdot & 0 \\
-\frac{c_{j}}{\sqrt{M m_{j}}} & 0 & 0 & \omega_{j}^{2}
\end{array}\right)
$$

The reaction co-ordinate $q$ may then be written as

$$
q=\sum_{j=0}^{N} U_{0 j} \eta_{i}
$$

Of the modes $\eta_{j}$, we take $\eta_{0}$ to be the unstable mode. We denote the frequency of $\eta_{j}$ as $\lambda_{j}$. $\eta_{0}$ has the imaginary frequency and we write it as $\lambda_{0}=i \Lambda$, where $\Lambda$ is real. As $\eta_{j}$ is a normal mode, its time development is given by

$$
\eta_{j}(t)=\eta_{0}(0) \cos \left(\lambda_{j} t\right)+p_{\eta_{j}}(0) \frac{\sin \left(\lambda_{j} t\right)}{\lambda_{j}}
$$

with $j=0,1,2, \ldots . N$.

Now the Hamiltonian when written in terms of the normal modes the coupling is no more there and is given by

$$
H_{n o r}=\sum_{j=0}^{N}\left(\frac{p_{j}^{2}}{2}+\frac{1}{2} \lambda_{j}^{2} \eta_{j}^{2}\right)
$$

In the next section we analytically derive the rate constant in the normal mode description using the above Hamiltonian.

\section{METHOD OF REACTIVE FLUX AND THE RATE CONSTANT}

In this section we briefly describe the method of reactive flux [7, 8] to calculate the rate constant. The method of reactive flux expresses the thermal rate constant (written in the mass-weighted coordinates) as

$$
k(t)=\frac{\left\langle\dot{q}(t) \delta(q(0)) \theta_{P}(q(t))\right\rangle}{\left\langle\theta_{R}(q(0))\right\rangle}=\frac{k_{n}(t)}{k_{d}(t)}
$$

where the angular bracket indicates the thermal average over the system as well as the bath degrees of freedom. The top of the barrier is chosen as $q(0)=0$. $\theta_{R}(q(0))$ is 1 if $q(0)<1$ and 0 otherwise. $\theta_{p}$ is just $1-\theta_{R}$, $\dot{q}$ is the velocity of the particle.

The numerator of Eq. (10) could be written as

$$
k_{n}(t)=-i\left(\frac{\partial}{\partial r_{0}}\left\langle\exp \left(i r_{0} \dot{q}(0)\right) \delta(q(0)) \theta_{P}(q(t))\right\rangle\right)_{r_{0}=0}
$$


Expressing the step function as an integral over delta function and using the Fourier integral representation of delta function the expression for $k_{n}(t)$ becomes

$$
k_{n}(t)=\left(\frac{1}{(2 \pi)^{2}} \int_{0}^{\infty} d h(-i) \frac{\partial}{\partial r_{0}} \int_{-\infty}^{\infty} d s_{0} \int_{-\infty}^{\infty} d s_{1} \exp \left(-i s_{1} h\right)\left\langle\exp \left(i r_{0} \dot{q}(0)+i s_{1} q(t)+i s_{0} q(0)\right)\right\rangle\right)_{r_{0}=0}
$$

The angular bracket indicates the thermal average over all degrees of freedom of the problem and it is done by multiplying the quantities within the angular bracket by $\exp \left(-\beta H_{\text {nor }}\right)$, and carrying out the integration over all coordinates and momenta degrees of freedom. Writing $k_{n}(t)$ in terms of $\eta_{j}$ leads to

$$
k_{n}(t)=\frac{\exp \left(-\beta E_{b}\right)}{(2 \pi)^{2}}\left(\begin{array}{c}
\int_{0}^{\infty} d h(-i) \frac{\partial}{\partial r_{0}} \int_{-\infty}^{\infty} d s_{0} \int_{-\infty}^{\infty} d s_{1} \exp \left(-i s_{1} h\right) \exp \left(-\beta\left(\frac{p_{\eta_{0}}^{2}}{2}-\frac{\Lambda^{2}}{2} \eta_{0}^{2}\right)\right) \exp \left(i r_{0} U_{00} p_{\eta_{0}}\right) \\
\exp \left(i r_{0} U_{00} \eta_{0}\right) \exp \left(i s_{1}\left(U_{00} \eta_{0} \cosh (\Lambda t)+p_{\eta_{0}}(0) \frac{\sinh (\Lambda t)}{\Lambda}\right)\right) \\
\prod_{j=1}^{N} \int_{-\infty}^{\infty} d p_{j} \int_{-\infty}^{\infty} d \eta_{j} \exp \left(-\beta\left(\frac{p_{j}^{2}}{2}+\frac{\lambda_{j}^{2}}{2} \eta_{j}^{2}\right)\right) \exp \left(i r_{0} U_{j 0} \eta_{j}\right) \exp \left(i r_{0} U_{j 0} p_{\eta_{j}}\right) \\
\exp \left(i s_{1}\left(U_{j 0} \eta_{j} \cos \left(\lambda_{j} t\right)+p_{\eta_{j}}(0) \frac{\sin \left(\lambda_{j} t\right)}{\lambda_{j}}\right)\right.
\end{array}\right)_{r_{0}=0}
$$

where $E_{b}$ is the barrier height. After one carries out the integrations the expression for $k_{n}(t)$ can be written as

$$
k_{n}(t)=\prod_{j=1}^{N}\left(\frac{2 \pi}{\beta \lambda_{j}}\right)\left(\frac{1}{\beta \Lambda}\right)\left(\frac{-\dot{c}(t)}{\left(c(t)^{2}-c(0)^{2}\right)^{\frac{1}{2}}}\right) \exp \left(-\beta E_{b}\right)
$$

Similarly the denominator of the Eq. (10) can be writ- ten as

$$
k_{d}(t)=\prod_{j=0}^{N} \int_{-\infty}^{\infty} d p_{j} \int_{-\infty}^{\infty} d \eta_{j} \exp \left(-\beta\left(\frac{p_{j}^{2}}{2}+\frac{\lambda_{j}^{0^{2}}}{2} \eta_{j}^{2}\right)\right)=\prod_{j=0}^{N}\left(\frac{2 \pi}{\beta \lambda_{j}^{0}}\right)
$$

Then the time dependent rate constant becomes

$$
k(t)=\frac{k_{n}(t)}{k_{d}(t)}=\frac{1}{2 \pi \Lambda} \frac{\prod_{j=0}^{N} \lambda_{j}^{0}}{\prod_{j=1}^{N} \lambda_{j}}\left(\frac{-\dot{c}(t)}{\left(c(t)^{2}-c(0)^{2}\right)^{\frac{1}{2}}}\right) \exp \left(-\beta E_{b}\right)
$$


Here we use the following identity [1], $\frac{\prod_{j=0}^{N} \lambda_{j}^{0}}{\Lambda \prod_{j=1}^{N} \lambda_{j}}=\frac{\omega_{0}}{\omega_{b}}$, and this simplifies our $k(t)$

$$
k(t)=\frac{1}{2 \pi} \frac{\omega_{0}}{\omega_{b}}\left(\frac{-\dot{c}(t)}{\left(c(t)^{2}-c(0)^{2}\right)^{\frac{1}{2}}}\right) \exp \left(-\beta E_{b}\right)
$$

where, $c(t)=\left(\sum_{j=0}^{N} \frac{U_{0 j}^{2} \cos \left(\lambda_{j} t\right)}{\lambda_{j}^{2}}\right)$. As usual $k(t)[16]$ is defined by

$$
k(t)=\kappa(t) k(0)
$$

We show in the Appendix that $k(0)=\frac{\omega_{0}}{2 \pi} \exp \left(-\beta E_{b}\right)$, which is nothing but the transition state rate constant $k_{T S T}$. In the Appendix we also show how one gets Kramers rate constant in the $t \rightarrow \infty$ limit.

Using the above expression for $k(0)$ our time dependent transmission coefficient, $\kappa(t)$ becomes

$$
\kappa(t)=\frac{1}{\omega_{b}}\left(\frac{-\dot{c}(t)}{\left(c(t)^{2}-c(0)^{2}\right)^{\frac{1}{2}}}\right)
$$

In the next section we calculate $c(t)$ and from that $\kappa(t)$ for Markovian as well as for Non Markovian noise.

\section{CALCULATION OF $c(t)$ AND $\kappa(t)$}

Calculation of $\kappa(t)$ involves the calculation of $c(t)$. First we describe a general derivation of $c(t)$ then we evaluate $\kappa(t)$ for different cases.

\section{A. Calculation of $c(t)$}

$c(t)$ is defined by $c(t)=\sum_{j=0}^{N} \frac{U_{j 0}^{2} \cos \left(\lambda_{j} t\right)}{\lambda_{j}^{2}}$. One proceeds by writing $\sum_{j=1}^{N} U_{j 0}^{2} \delta\left(\omega^{2}-\lambda_{j}^{2}\right)=-\frac{1}{\pi} \operatorname{Im} \sum_{j=1}^{N} \frac{U_{j 0}^{2}}{\left(\omega^{2}+i \eta-\lambda_{j}^{2}\right)}$. To evaluate $\sum_{j=1}^{N} \frac{U_{j 0}^{2}}{\left(\omega^{2}+i \eta-\lambda_{j}^{2}\right)}$ here we adopt notations similar to quantum mechanics and also use the fact that $D$ is a matrix with eigen values $\lambda_{j}^{2}, j=1,2, \ldots . N$.

$$
\sum_{j=1}^{N} \frac{U_{j 0}^{2}}{\left(\omega^{2}+i \eta-\lambda_{j}^{2}\right)}=\sum_{j=1}^{N}\langle 0 \mid j\rangle \frac{1}{\left(\omega^{2}+i \eta-\lambda_{j}^{2}\right)}\langle j \mid 0\rangle=\sum_{j=1}^{N}\langle 0 \mid j\rangle \frac{1}{\left(\omega^{2}+i \eta-D\right)}\langle j \mid 0\rangle
$$

Then by introducing $|j\rangle\langle j|$ in between the sum and using the resolution of identity $\sum_{j=1}^{N}|j\rangle\langle j|=I$.

$$
\sum_{j=1}^{N} U_{j 0}^{2} \delta\left(\omega^{2}-\lambda_{j}^{2}\right)=\left\langle 0\left|\frac{1}{\left(\omega^{2}+i \eta-D\right)}\right| 0\right\rangle=-\frac{1}{\pi} \operatorname{Im}\left(\underline{\underline{G}}\left(\omega^{2}+i \eta\right)\right)
$$

Using partitioning technique one can evaluate $(\underline{\underline{G}})_{00}$ to get

$$
\begin{gathered}
\left.\underline{\underline{G}}\left(\omega^{2}+i \eta\right)\right)_{00} \\
=\left(\omega^{2}+\omega_{b}^{2}-\frac{1}{M} \sum_{j=1}^{N} \frac{c_{j}^{2} \omega^{2}}{m_{j} \omega_{j}^{2}\left(\omega^{2}+i \eta-\omega_{j}^{2}\right)}\right)^{-1}
\end{gathered}
$$

Then using the definition of $J(\omega)$ Eq. (6) it becomes

$$
\begin{gathered}
\left(\underline{\underline{G}}\left(\omega^{2}+i \eta\right)\right)_{00} \\
=\left(\omega^{2}+\omega_{b}^{2}-\frac{2 \omega^{2}}{M \pi} \int_{0}^{\infty} \frac{J(\tilde{\omega}) d \tilde{\omega}}{\tilde{\omega}\left(\omega^{2}+i \eta-\tilde{\omega}^{2}\right)}\right)^{-1}
\end{gathered}
$$

$$
\begin{aligned}
& \operatorname{As}-\frac{1}{\pi} \operatorname{Im}\left(\underline{\underline{G}}\left(\omega^{2}\right)\right)_{00}=\sum_{j=0}^{N} U_{j 0}^{2} \delta\left(\omega^{2}-\lambda_{j}^{2}\right)=\rho\left(\omega^{2}\right) . \mathrm{We} \\
& \text { can write } c(t)=2 \int_{0}^{\infty} \frac{d \omega}{\omega} \cos (\omega t) \rho\left(\omega^{2}\right) .
\end{aligned}
$$

B. Calculation of $\kappa(t)$ with $\gamma(t)=\gamma 2 H(2 H-1)|t|^{2 H-2}$

First we perform the calculation with the time dependent friction $\gamma(t)$ used by Xie [21], which is 


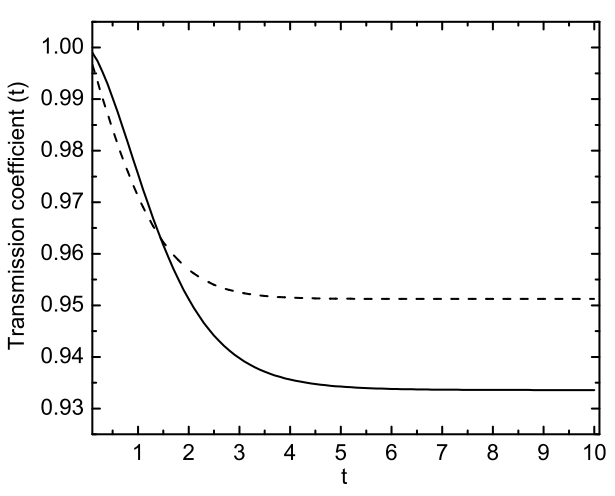

FIG. 1: Time dependent transmission coefficient, $\kappa(t)$ against time, for $H=\frac{1}{2}$ (dashed line) and $H=\frac{3}{4}$ (solid line), parameters used are $\omega_{b}=1, \gamma=0.1$

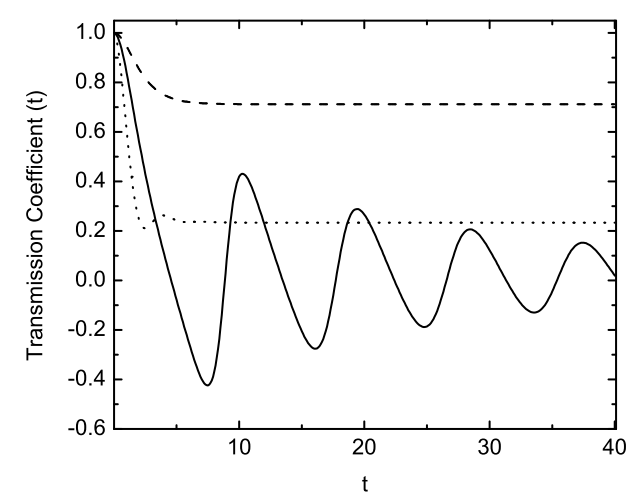

FIG. 2: Time dependent transmission coefficient, $\kappa(t)$ against time for Non-Markovian friction, $\gamma(t)=\gamma h \exp (-h t)$. Three different regimes, caging regime (solid line) with parameters, $\gamma=150, h=0.01, \omega_{b}=1$, intermediate regime (dotted line) with parameters $\gamma=5, h=1, \omega_{b}=1$, and nonadiabatic regime (dashed line) with parameters $\gamma=50, h=0.01, \omega_{b}=$ 1.

$$
\gamma(t)=\gamma 2 H(2 H-1)|t|^{2 H-2}
$$

with, $\frac{1}{2}<H<1$, thus the thermal fluctuations are described by fractional Gaussian noise [21]. Now we take a inverse Fourier transform of Eq. (5) and write $J(\omega)$ in terms of $\gamma(t)$.

$$
J(\omega)=M \omega \int_{0}^{\infty} d t \gamma(t) \cos (\omega t)
$$

Using the definition of $\gamma(t)$ we get

$$
\frac{2 \omega^{2}}{M \pi} \int_{0}^{\infty} \frac{J(\tilde{\omega}) d \tilde{\omega}}{\tilde{\omega}\left(\omega^{2}+i \eta-\tilde{\omega}^{2}\right)}=\gamma \Gamma(2 H+1) \Lambda^{2-2 H}
$$

First we consider the case $\omega^{2}=-\Lambda^{2}$ which means we consider the unstable mode of the problem. Then

$$
\left(\underline{\underline{G}}\left(-\Lambda^{2}\right)\right)_{00}=\left(-\Lambda^{2}+\omega_{b}^{2}-\gamma \Gamma(2 H+1) \Lambda^{2-2 H}\right)^{-1}
$$

Our next task is to find out the poles, i.e. the unstable modes for which $G_{00}\left(-\Lambda^{2}\right)$ blows up, i.e. the solutions of the equation

$$
-\Lambda^{2}+\omega_{b}^{2}-\gamma \Gamma(2 H+1) \Lambda^{2-2 H}=0
$$

On the other hand, to find $\rho\left(\omega^{2}\right)$ we have to consider a situation where $\omega^{2}$ is positive. In other words the stable modes. Now the Evaluation of $\rho\left(\omega^{2}\right)$ involves the inte$\operatorname{gral} \frac{2 \omega^{2}}{M \pi} \int_{0}^{\infty} \frac{J(\tilde{\omega}) d \tilde{\omega}}{\tilde{\omega}\left(\omega^{2}+i \eta-\tilde{\omega}^{2}\right)}$. Using the identity $\frac{1}{\left(\omega^{2}+i \eta-\tilde{\omega}^{2}\right)}=$ $P\left(\frac{1}{\omega^{2}-\tilde{\omega}^{2}}\right)-i \pi \delta\left(\omega^{2}-\tilde{\omega}^{2}\right)$, where $P\left(\frac{1}{\omega^{2}-\tilde{\omega}^{2}}\right)$ is the principal value of $\left(\frac{1}{\omega^{2}-\tilde{\omega}^{2}}\right)$ we find

$\frac{2 \omega^{2}}{M \pi} \int_{0}^{\infty} \frac{J(\tilde{\omega}) d \tilde{\omega}}{\tilde{\omega}\left(\omega^{2}+i \eta-\tilde{\omega}^{2}\right)}=-\exp (i H \pi) \Gamma(2 H+2) \omega^{2-2 H} \gamma$

Thus

$$
\left(\underline{\underline{G}}\left(\omega^{2}\right)\right)_{00}=\left(\omega^{2}+\omega_{b}^{2}+\exp (i H \pi) \Gamma(2 H+2) \omega^{2-2 H} \gamma\right)^{-1}
$$

and $\downarrow$

$$
\begin{gathered}
\rho\left(\omega^{2}\right)=-\frac{1}{\pi} \operatorname{Im}\left(\underline{\underline{G}}\left(\omega^{2}\right)\right)_{00} \\
=\frac{1}{\pi} \frac{\gamma \Gamma(2 H+1) \omega^{2-2 H} \sin (H \pi)}{\left(\left(\omega^{2}+\omega_{b}^{2}+\gamma \Gamma(2 H+1) \omega^{2-2 H} \cos (H \pi)\right)^{2}+\gamma^{2} \sin ^{2}(H \pi) \Gamma(2 H+1)^{2} \omega^{4-4 H}\right)}
\end{gathered}
$$




\section{The Case $H=\frac{1}{2}$}

First we consider $H=\frac{1}{2}$, for which the $\gamma(t)=2 \gamma \delta(t)$ and with this the noise-noise correlation function defined in Eq. (7) becomes delta function correlated, reading

$$
\left\langle\zeta\left(t_{1}\right) \zeta\left(t_{2}\right)\right\rangle=2 M \gamma k_{B} T \delta\left(t_{1}-t_{2}\right)
$$

Then the dynamics is described by usual Langevin equation rather than a GLE. Thus the dynamics is then Markovian i.e. with no memory. For the Markovian case Eq. (26) becomes, $-\Lambda^{2}+\omega_{b}^{2}-\gamma \Lambda=0$ and has two roots. But only one of them is physically acceptable. The root is, $\Lambda_{H=\frac{1}{2}}=-\frac{\gamma}{2}+\sqrt{\left(\frac{\gamma}{2}\right)^{2}+\omega_{b}^{2}}$. Similarly from Eq. (29) one gets $\rho\left(\omega^{2}\right)_{H=\frac{1}{2}}=\frac{\omega \gamma}{\pi\left(\omega^{2} \gamma^{2}+\left(\omega^{2}+\omega_{b}^{2}\right)^{2}\right)}$. Then $\Lambda_{H=\frac{1}{2}}$ and $\rho\left(\omega^{2}\right)_{H=\frac{1}{2}}$ are used to calculate $c(t)$ and taking $t \rightarrow 0$ limit one gets $c(0)$. In this case we could do these analytically. The time dependent transmission coefficient in this case is given by

$$
\kappa(t)=\frac{\left(-1+e^{t(f-\gamma)}\right)}{\sqrt{\left(1-4 e^{f t}+2 e^{t(f-\gamma)}+2 e^{2 t(f-\gamma)}\right)-\left(\frac{\gamma}{2 \omega_{b}}\right)\left(f-f e^{2 t(f-\gamma)}+2 \gamma\left(-1+e^{f t}\right)\right)}}
$$

where $f=\gamma+\left(4 \omega_{b}^{2}+\gamma\right)^{1 / 2}$.

$\kappa(t)$ is then calculated using the above expression and plotted against time (Fig. 1).

$$
\text { 2. The Case } H=\frac{3}{4}
$$

When $H \neq \frac{1}{2}$ then the noise is no longer delta function correlated, i.e. not a white noise with Gaussian distribution. This regime is know as Non Markovian regime which has memory. We have similarly evaluated $\kappa(t)$ for $H=\frac{3}{4}$ and plotted against time in Fig. 2. But in this case the integrations are done numerically since analytical evaluation of integrals were not possible.

\section{Calculation of $\kappa(t)$ with $\gamma(t)=\gamma h \exp (-h t)$}

In this section we carry out the calculation of $\kappa(t)$ with a time dependent friction $\gamma(t)=\gamma h \exp (-h t)$. The same friction is used by Kohen and Tannor [16, 17]. First we calculate $J(\omega)$ with this friction as follows

$$
J(\omega)=M \gamma h \omega \int_{0}^{\infty} \exp (-h t) \cos (\omega t) d t=\frac{\gamma M h^{2} \omega}{\left(h^{2}+\omega^{2}\right)}
$$

Then the following integral is evaluated as follows

$$
\frac{2 \omega^{2}}{M \pi} \int_{0}^{\infty} \frac{J(\tilde{\omega}) d \tilde{\omega}}{\left(\alpha^{2}+\tilde{\omega}^{2}\right)\left(\omega^{2}+i \eta-\tilde{\omega}^{2}\right)}=\frac{2 \omega^{2} h^{2}}{\pi} \int_{0}^{\infty} \frac{d \tilde{\omega}}{\left(\alpha^{2}+\tilde{\omega}^{2}\right)\left(\omega^{2}+i \eta-\tilde{\omega}^{2}\right)}=\frac{\gamma h \omega(\omega-i h)}{\left(h^{2}+\omega^{2}\right)}
$$

We have used the identity, $\frac{1}{\left(\omega^{2}+i \eta-\tilde{\omega}^{2}\right)}=P\left(\frac{1}{\omega^{2}-\tilde{\omega}^{2}}\right)-\quad$ and $i \pi \delta\left(\omega^{2}-\tilde{\omega}^{2}\right)$, where $P\left(\frac{1}{\omega^{2}-\tilde{\omega}^{2}}\right)$ is the principal value of $\left(\frac{1}{\omega^{2}-\tilde{\omega}^{2}}\right)$. Thus

$$
\left(\underline{\underline{G}}\left(\omega^{2}\right)\right)_{00}=\left(\omega^{2}+\omega_{b}^{2}-\frac{\gamma h \omega(\omega-i h)}{\left(h^{2}+\omega^{2}\right)}\right)^{-1}
$$

$$
\rho\left(\omega^{2}\right)=-\frac{1}{\pi} \operatorname{Im}\left(\underline{\underline{G}}\left(\omega^{2}\right)\right)_{00}=\frac{\omega \gamma h^{2}\left(h^{2}+\omega^{2}\right)}{\pi\left(\left(\left(h^{2}+\omega^{2}\right)\left(h^{2}+\omega_{b}^{2}\right)-\gamma h \omega^{2}\right)^{2}+\omega^{2} \gamma^{2} h^{4}\right)}
$$


One should notice that

$$
\lim _{h \rightarrow \infty} \frac{\omega \gamma h^{2}\left(h^{2}+\omega^{2}\right)}{\pi\left(\left(\left(h^{2}+\omega^{2}\right)\left(h^{2}+\omega_{b}^{2}\right)-\gamma h \omega^{2}\right)^{2}+\omega^{2} \gamma^{2} h^{4}\right)}=\frac{\gamma \omega}{\pi\left(\left(\omega^{2}+\omega_{b}^{2}\right)+\gamma^{2} \omega^{2}\right)}=\rho\left(\omega^{2}\right)_{H=\frac{1}{2}}
$$

Thus in this limit the result obtained is identical with the friction $\gamma(t)=\gamma 2 H(2 H-1)|t|^{2 H-2}$ with $H=\frac{1}{2}$. In other words the Markovian dynamics is recovered.

To calculate $\kappa(t)$ we proceed as in the previous case.

First considering the case $\omega^{2}=-\Lambda^{2}$, one gets

$$
\frac{2 \omega^{2}}{M \pi} \int_{0}^{\infty} \frac{J(\tilde{\omega}) d \tilde{\omega}}{\left(\alpha^{2}+\tilde{\omega}^{2}\right)\left(\omega^{2}+i \eta-\tilde{\omega}^{2}\right)}=\frac{\gamma h \Lambda}{(h+\Lambda)}
$$

Thus

$$
\left(\underline{\underline{G}}\left(\omega^{2}\right)\right)_{00}=\left(-\Lambda^{2}+\omega_{b}^{2}-\frac{\gamma h \Lambda}{(h+\Lambda)}\right)^{-1}
$$

Hence to find out the poles one has to solve the solution of the equation

$$
-\Lambda^{2}+\omega_{b}^{2}-\frac{\gamma h \Lambda}{(h+\Lambda)}=0
$$

To explore the non-Markovian dynamics we choose three set of parameters corresponding to three different regimes of non-Markovian dynamics following Kohen and Tannor [16, 17]. These regimes are called non-adiabatic, caging and intermediate according to Kohen and Tannor [16, 17]. The time dependent coefficients in these regimes are calculated numerically and plotted against time. We observe similar kind of oscillatory behavior of $\kappa(t)$ in the caging regime as obtained by them. Similarly in the intermediate regime the plot looks similar with a nonmonotonic decay (Fig. 2).

\section{CONCLUSIONS}

The paper shows an elegant way of combining the traditional system plus reservoir model [9, 10] and the method of reactive flux [7, 8] to calculate the time dependent transmission coefficient, $\kappa(t)$. Analytically we derive a general formula for $\kappa(t)$ Eq. (18). For the Markovian case with no memory $\left(H=\frac{1}{2}\right)$ for the friction $\gamma(t)=\gamma 2 H(2 H-1)|t|^{2 H-2}$ with $H=\frac{1}{2}$ which is same as with $h=\infty$ for the friction $\gamma(t)=\gamma h \exp (-h t)$, $\kappa(t)$ is calculated analytically. Whereas in the case with memory $\left(H=\frac{3}{4}\right)$ and with any finite value of $h$ the calculations were done numerically. In all the cases a plot of $\kappa(t)$ vs $t$ starts from 1 at $t=0$ and reaches a plateau in the long time limit. As expected our formula for the time dependent rate constant, $k(t)$ becomes equal to the transition state rate constant when one takes the limit $t \rightarrow 0$ [16, 17]. Similarly Kramers rate constant is obtained by taking $t \rightarrow \infty[16,17]$. In future we would like to extend our formulation to quantum domain.

\section{ACKNOWLEDGEMENTS}

The author is grateful to Prof. K. L. Sebastian for his useful comments and encouragements and thanks Prof. B. J. Cherayil for his comments on the manuscript. The author also acknowledges Council of Scientific and Industrial Research (CSIR), India for financial support.

\section{APPENDIX : TRANSITION STATE RATE AND KRAMERS RATE FROM $k(t)$}

First we take the limit $t \rightarrow 0$. Then from Eq. (16) one writes 


$$
\begin{gathered}
k(0)=\lim _{t \rightarrow 0} k(0+t)=\frac{1}{2 \pi} \frac{\omega_{0}}{\omega_{b}} \lim _{t \rightarrow 0} \frac{-\dot{c}(0+t)}{\left(c(0+t)^{2}-c(0)^{2}\right)^{\frac{1}{2}}} \exp \left(-\beta E_{b}\right)= \\
\frac{1}{2 \pi} \frac{\omega_{0}}{\omega_{b}} \lim _{t \rightarrow 0} \frac{-\dot{c}(0)-\ddot{c}(0) t}{\left(\left(c(0)+t \dot{c}(0)+\frac{t^{2}}{2} \ddot{c}(0)\right)^{2}-c(0)^{2}\right)^{\frac{1}{2}}} \exp \left(-\beta E_{b}\right) \\
=\frac{1}{2 \pi} \frac{\omega_{0}}{\omega_{b}} \lim _{t \rightarrow 0} \frac{-\ddot{c}(0) t}{\left(c(0)^{2}-c(0)^{2}+c(0) \ddot{c}(0) t^{2}\right)^{\frac{1}{2}}} \exp \left(-\beta E_{b}\right)=\frac{1}{2 \pi} \frac{\omega_{0}}{\omega_{b}} \frac{-\ddot{c}(0)}{\sqrt{c(0) \ddot{c}(0)}} \exp \left(-\beta E_{b}\right)=\frac{1}{2 \pi} \frac{\omega_{0}}{\omega_{b}} \frac{1}{\sqrt{-c(0)}} \exp \left(-\beta E_{b}\right)
\end{gathered}
$$

Where we have used the facts, $\dot{c}(0)=0$ and $\ddot{c}(0)=-1$ . This is because $\dot{c}(t)=-2 \int_{0}^{\infty} d \omega \rho\left(\omega^{2}\right) \sin (\omega t)$ making $\dot{c}(0)=0 . \quad$ Similarly $\ddot{c}(t)=-2 \int_{0}^{\infty} d \omega \rho\left(\omega^{2}\right) \sin (\omega t) \omega=$
$-\sum_{j=0}^{N} U_{j 0}^{2}=-1$. Now one can also show, $-c(0)=\frac{1}{\omega_{b}^{2}}$ as follows

$$
\begin{gathered}
c(0)=\sum_{j=0}^{N} \frac{U_{j 0}^{2}}{\lambda_{j}^{2}}=\sum_{j=0}^{N} U_{j 0} \lambda_{j}^{-2} U_{0 j}=\sum_{j=0}^{N}\langle 0 \mid j\rangle \lambda_{j}^{-2}\langle j \mid 0\rangle \\
=-\lim _{\omega^{2} \rightarrow 0} \sum_{j=0}^{N}\langle 0 \mid j\rangle\left\langle j\left|\frac{1}{\left(\omega^{2}-D\right)}\right| j\right\rangle\langle j \mid 0\rangle=-\lim _{\omega^{2} \rightarrow 0} \sum_{j=0}^{N}\left\langle 0\left|\frac{1}{\left(\omega^{2}-D\right)}\right| 0\right\rangle=-\lim _{\omega^{2} \rightarrow 0}\left(\underline{\underline{G}}\left(\omega^{2}\right)\right)_{00}
\end{gathered}
$$

From Eq. (22) one can see that $\lim _{\omega^{2} \rightarrow 0}\left(\underline{\underline{G}}\left(\omega^{2}\right)\right)_{00}=$ $\frac{1}{\omega_{b}^{2}}=-c(0)$. Then $k(0)$ is nothing but the transition

Similarly taking $t \rightarrow \infty$ one gets the Kramers rate constant as follows state rate constant, $k_{T S T}$.

$$
k(0)=\frac{\omega_{0}}{2 \pi} \exp \left(-\beta E_{b}\right)=k_{T S T}
$$

$$
k(\infty)=\frac{1}{2 \pi} \frac{\omega_{0}}{\omega_{b}}\left(\frac{\frac{U_{00}^{2}}{\Lambda} \sinh (\Lambda t)}{\sqrt{\left(\frac{U_{00}^{2}}{\Lambda^{2}} \cosh (\Lambda t)\right)^{2}}}\right)_{t \rightarrow \infty} \exp \left(-\beta E_{b}\right)=\frac{1}{2 \pi} \frac{\omega_{0}}{\omega_{b}}(\Lambda \tanh (\Lambda t))_{t \rightarrow \infty} \exp \left(-\beta E_{b}\right)
$$

Then

$$
\begin{gathered}
k(\infty)=\Lambda \frac{1}{2 \pi} \frac{\omega_{0}}{\omega_{b}} \exp \left(-\beta E_{b}\right) \\
k(\infty)=\left(-\frac{\gamma}{2}+\sqrt{\left(\frac{\gamma}{2}\right)^{2}+\omega_{b}^{2}}\right) \frac{1}{2 \pi} \frac{\omega_{0}}{\omega_{b}} \exp \left(-\beta E_{b}\right)
\end{gathered}
$$

Now if one replaces $\Lambda$ by $\Lambda_{H=\frac{1}{2}}=-\frac{\gamma}{2}+\sqrt{\left(\frac{\gamma}{2}\right)^{2}+\omega_{b}^{2}}$ ( $H=\frac{1}{2}$ means white noise) one gets

Which is nothing but the usual Kramers rate. Also in the overdamped limit i.e. when $\gamma>>\omega_{b}^{2}$ one gets 


$$
k(\infty)=\left(-\frac{\gamma}{2}+\frac{\gamma}{2}\left(1+\frac{1}{2} 4\left(\frac{\omega_{b}^{2}}{\gamma^{2}}\right)\right)\right) \frac{\omega_{0}}{2 \pi \omega_{b}} \exp \left(-\beta E_{b}\right)=\frac{\omega_{0} \omega_{b}}{2 \pi \gamma} \exp \left(-\beta E_{b}\right)
$$

which is Kramers rate expression in overdamped regime.

[1] P. Hänggi, P. Talkner and M. Borkovec, Rev. Mod. Phys. 62, 251 (1990).

[2] A. Nitzan, Chemical Dynamics in condensed phases (Oxford University Press, New York, 2006).

[3] H. A. Kramers, Physica (Amsterdam) 7, 284 (1940).

[4] W. T. Coffey, Y. P. Kalmykov, and J. T. Waldron, The Langevin Equation, Second Edition (World Scientific, 2004).

[5] R. F. Grote and J. T. Hynes, J. Chem. Phys. 73, 2715 (1980).

[6] P. Hänggi and F. Mojtabai, Phys. Rev. A 26, 1168 (1986).

[7] D. Chandler, J. Stat. Phys. 42, 49 (1986).

[8] D. Chandler, Introduction to Modern Statistical Mechanics (Oxford University Press, New York, 1987).

[9] U. Weiss, Quantum Dissipative Systems (World Scientific, 1999).

[10] R. Zwanzig, Nonequilibrium Statistical Mechanics (Oxford University Press, New York, 2001).

[11] H. Zhou and R. Zwanzig, J. Phys. Chem. A 106, 7562
(2002).

[12] W. Min and X. S. Xie, Phys. Rev. E 73, 010902 (2006).

[13] A. M. Levin, M. Shapiro and E. Pollak, J. Chem. Phys. 88, 1959 (1988).

[14] E. Pollak, J. Chem. Phys. 85, 865 (1986).

[15] E. Pollak, H. Grabert, and P. Hänggi, J. Chem. Phys. 91, 4073 (1989).

[16] D. Kohen and D. J. Tannor, J. Chem. Phys. 103, 6013 (1995).

[17] D. Kohen and D. J. Tannor, Adv. Chem. Phys. 111, 219 (2000).

[18] J. Bao, J. Chem. Phys. 124, 114103 (2006).

[19] S. Chowdhury and B. J. Cherayil, J. Chem. Phys. 125, 024904 (2006).

[20] D. Barik, S K. Banik and D. S. Ray, J. Chem. Phys. 119, 680 (2003).

[21] S. C. Kou and X. S. Xie, Phys. Rev. Lett. 93, 180603 (2004). 